\title{
Research on Enterprise Financing Risk Assessment Based on Analytic Hierarchy Process
}

\author{
Ya-Qiong PAN ${ }^{1, a}$, Xiao-Qin ZHANG ${ }^{2, b}$ \\ School of Management, Wuhan University of Science and Technology, Wuhan 430081, China \\ apanyaqiong2002@sina.com, ${ }^{\mathrm{b}} 491079422 @ q q . c o m$
}

Keywords: Enterprise financing, Risk assessment, Analytic hierarchy process.

\begin{abstract}
With the continuous development of economy, the level and scale of enterprise financing has increased rapidly in China. Meanwhile, enterprises also face various financing risks. This paper discusses the common approaches of corporate financing, and other potential risks. The Analytic Hierarchy Process is designed to make risk quantification analysis by considering macro factors, medium industry factors and micro enterprise factors. The findings are shown that laws and regulations limits, national macroeconomic regulation and control policy have largest impact on financing risks.
\end{abstract}

\section{Introduction}

Enterprise financing risk mainly refers to the possibility of losing solvency and the variability of profits when enterprises finance (Wang Jianpu, 2011) [1]. It gradually becomes a speculative risk which may bring opportunities and benefits, but also may cause hidden threats and the risk of loss. Enterprise financing risk degree is more complicated than it used to be. It faces greater uncertainty in the fierce market competition. Therefore, it is imperative to research and analyze the risk factors in the process of enterprise financing.

\section{Literature Reviews}

There are a lot of literatures to explore the importance of financing risk management, assessment content and financing method overseas. Xiangjuan Tang (2012) thought that the financing risk is the biggest bottleneck restricting the development of small and medium-sized enterprises. In the era of post financial crisis, financing risk management should be strengthened[2]. Yede Huang (2010) argued that corporate financing is crucial as the starting point of the production operation, and it is faced with a variety of financing risk mainly including external environment factors and internal management factors. Enterprise financing has two kinds of ways by internal financing and external financing [3]. Myers \& Majluf (1984) considered that according to the optimal sequence financing theory, the internal financing may be best preferred due to the lowest costs, then the second choice is debt financing, and the last choice is equity financing. In addition, there is another way of financing, which is mixed financing[4].

Some domestic literatures mainly summarize enterprise types and influencing factors of financing risk. Zhang Anping (2010) analyzed from the angle of information asymmetry and found that adverse selection and moral hazard is the main reason for the higher risk of financing[5]. Du Xiumin (2010) summed up small and medium-sized enterprise financing risks which are composed by undiversifiable risk and diversifiable risk. Undiversifiable risk includes the risk of national policy and market changes. Diversifiable risk includes operation, finance, management, capital and credit risk[6].Wang Yanlin (2012) thought that the major factors affect small and medium-sized enterprise the financing risk which are composed of free cash flow, profit ability, operation ability, solvency, enterprise size, equity structure and so on. Research results found that small and medium-sized enterprise financing risk has a significantly positive correlation with the former three factors, and do not significantly correlated with the equity structure, while has a significantly negative correlation with other factors[7]. Li Suhong (2013) explored influence factors of financing risk from two external ways which are debt and equity financing. Then a risk evaluation model was built and conclusion was drawn that macro policy and law has the greatest effect on financing risk, while micro enterprise credit situation, financial status and enterprise operating management factors have the greater influences[8]. So it can be concluded that influenced 
factors of enterprises financing risk mainly include macro factors, medium-industry factors and micro enterprise factors. Through analyzing these factors, some advices can be taken to reduce the risk of financing for policymakers.

There are many kinds of financing risk assessment methods. Li X \& Zhang R (2013) used fuzzy analytic hierarchy process and fuzzy mathematics theory, set risk evaluation index system of pledge risk, financing enterprise credit risk, logistics enterprise risk and regulatory risk. When bank and enterprise information asymmetry brings a certain risk, it may conduct risk assessment at bank logistics and financial business [9]. Fan Xiaojun (2004) proposed financing risk evaluation index for large infrastructure project, conducted quantitative research on financing risk with a dynamic view by using fuzzy method[10].Guo Jie (2009) chose quantitative financial indicators and qualitative non-financial indicators, using efficacy coefficient method and analytic hierarchy process to evaluate enterprise financing risk comprehensively[11].Zhao Fabin (2013) analyzed financing channel and risks of practical training equipment, which mainly include credit, equipment losses, operation and interest rate risk, and concluded maximum risk of loans by using the analytic hierarchy process[12].

This paper takes corporate financing risk assessment as a research object, considering three different angles of enterprise financing risks which consist of macro factors, medium industry factors and micro enterprise factors. Analyze and rank the major risks during enterprise financing by using analytic hierarchy process, propose rationalization suggestions on how to choose appropriate financing ways and provide a basis for decision making.

\section{Enterprise Financing Risk Evaluation System}

In this paper, we quantitatively analyses the influence on enterprise financing risk, using analytic hierarchy process to establish the comprehensive evaluation system. Analytic hierarchy process is a simple, flexible and practical multi-criteria decision making method to analyze quantitatively on qualitative problems. Then the first-level indicators including bond financing, equity financing and mixed financing are summarized, which consists of some secondary and tertiary indicators.

The first target layer of financing risk evaluation system diagram is enterprise financing risk; The second criterion layer is bond financing, equity financing and mixed financing; The third criterion layer includes bank loans, issuing bonds and private lending in the debt financing as the main way of enterprise financing, public financing and asset restructuring for equity financing way, convertible securities and warrants for mixed financing way; The fourth layer scheme involves legal restrictions, current economic situation, national macroeconomic regulation and control policy, benefit compensation mechanism, monopoly and competition between the industry, industry threshold limit, profit ability, operation ability, solvency, enterprise size, etc. Due to the endogenous financing risk is relatively small when evaluating domestic enterprise financing risk, external oriented organizational structure should be adopted preferentially. Therefore, this paper just digs the financing risk of enterprises in exogenous financing way. The financing risk evaluation system is shown as Fig 1.

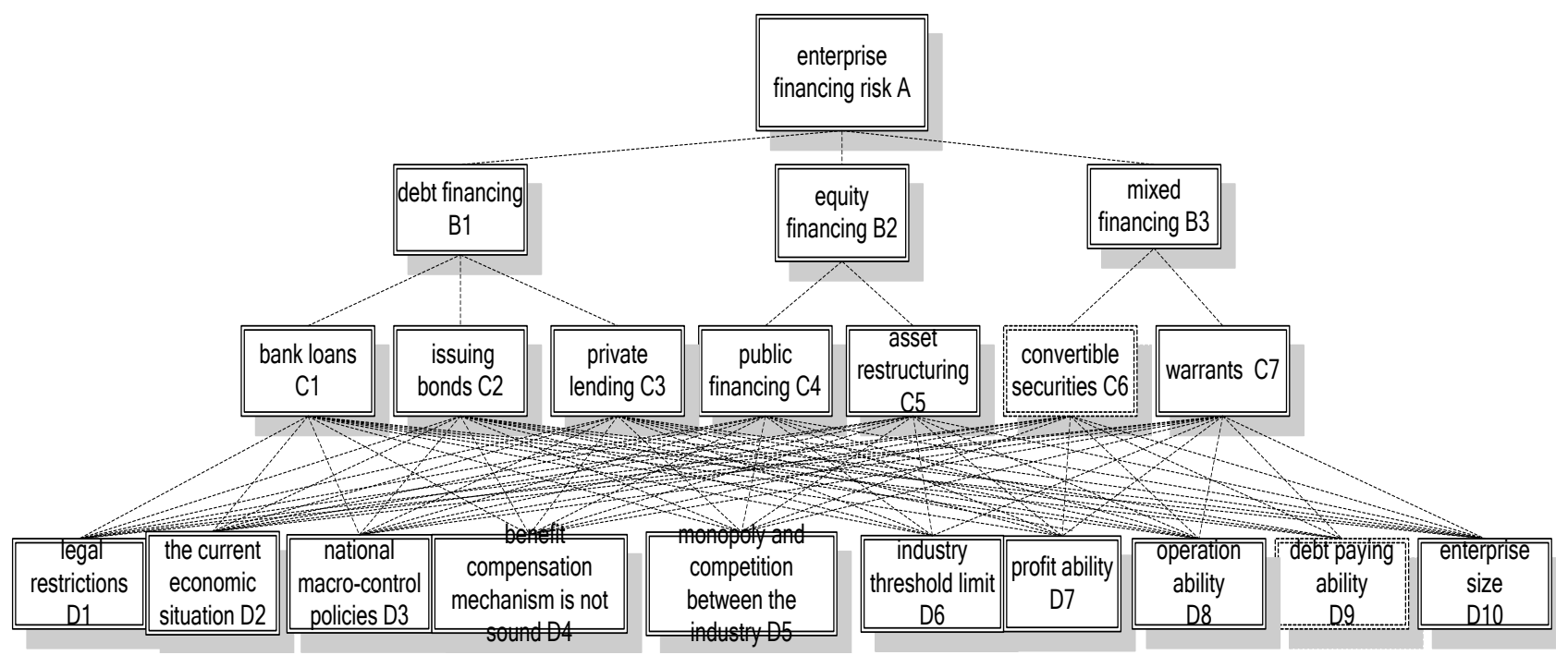

Fig.1 Financing risk evaluation system 
To expound the basic process of analytic hierarchy process being used for risk management, for example, we can figure out that how to choose the least risky way from bank loans, issuing bonds, private lending, public financing and asset restructuring, convertible securities and warrants and other common enterprise financing way when enterprises consider bond financing, equity financing and mixed financing.

\section{Enterprise Financing Risk Assessment Model}

Adopting analytic hierarchy process, parameters are estimated, analysis matrix is constructed and then weights are calculated. The main processes of determining weights: apply judgment matrix method to calculate the weight of each index weight; constitutes a decision on the issue of indicators to establish multi-hierarchical model; seek expert advice on the same level indicators pairwise comparisons and determine their relative importance according to assessment scales to determine the judgment matrix; integrate importance calculations to determine the weight of each index.

\section{Weight determination}

Here the weight coefficient matrix $r$ is determined by expert judgment and analytic Hierarchy Process matrix is derived. Then $s$ sub-goals and $t$ indicators of weight are drawn, and consistency check is tested. Finally, the weights in the enterprise credit evaluation model are obtained. The process is shown as follows:

1). Seven experts are invited to compare s sub-goals in the evaluation system with the various indicators of sub-goals respectively using analytic hierarchy process. Then several judging matrix was obtained. Here, the letter $a$ represents the number of experts, the letter $m$ for sub-goals or the number of indicators in the sub-goals; the type $T_{i j}$ for the judgment coefficient of the index $i$ and index $j$ in Eq. 1 .

$$
A_{a}=\left(T_{i j}\right)
$$

2). Construct judgment matrix

According to the principle of clustering analysis, filtrate and correct the assessment of seven experts' weighting using the analytic hierarchy process, to make the expert group evaluation index weight results conform to the majority principle of mathematics. Relative to the enterprise financing risk, compare the relative importance of each criterion. (judgment matrix A-B)

$$
b=B 2\left[\begin{array}{ccc}
1 & 3 & 5 \\
1 / 3 & 1 & 3 \\
1 / 5 & 1 / 3 & 1
\end{array}\right]^{B 3}
$$

Relative to the bond financing B1, equity financing B2 and mixed financing B3, to compare the relative importance of each criterion, the judgment matrixes were shown as the B1-C, B2-C and B3- C respectively.

$$
c_{1}=C 2\left[\begin{array}{ccc}
1 & 2 & 2 \\
1 / 2 & 1 & 2 \\
1 / 2 & 1 / 2 & 1
\end{array}\right] \quad \quad \quad \quad c_{2}={ }_{C 5}^{C 4}\left[\begin{array}{cc}
C 4 & C 5 \\
1 & 2 \\
1 / 2 & 1
\end{array}\right] \quad c_{3}={ }_{C 7}^{C 6}\left[\begin{array}{ccc}
C 6 & C 7 \\
1 / 2 & 1
\end{array}\right]
$$

Compared to bank loans, issuing bonds, private lending, public financing and asset restructuring, convertible securities and warrants, to compare the relative importance of each scheme layer, the judgment matrixes are the C1-D, C2-D, C3-D, C4-D, C5-D, C6-D and C7-D respectively. Due to space limited, these matrixes are omitted here.

3). Eigenvector W

Eigenvector $W$ is equivalent to seek eigenvector problem of judgment matrix A, that is the solution vector $\mathrm{W}$ of $A W=\lambda_{\max } W$. If the given judgment coefficient by expert is correct, then we get the maximum eigenvalue $\lambda$ max of judgment matrix $\mathrm{A}$ and the corresponding feature vector $\mathrm{W}$. 
The relative importance weights $W$ of judgment matrix A-B and $\lambda \max , \mathrm{CR}: W=\left[\begin{array}{ll}0.9161 & 0.3715\end{array}\right.$ $0.1506]^{\mathrm{T}} ; \lambda \max =3.0385 ; C R=0.0332$

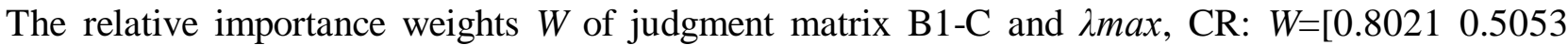
$0.3183]^{T} ; \lambda_{\max }=3.0536 ; C R=0.0462$

The relative importance weights $W$ of judgment matrix B2-C and B3-C and $\lambda$ max, CR: $\mathrm{W}=[0.8944$ $0.4472]^{T} ; \lambda_{\max }=2 ; C I=0$

The relative importance weights $W$ of judgment matrix C1-D and $\lambda$ max, CR: $W=[0.76570 .23480 .4815$ $0.14770 .19050 .16580 .11710 .11710 .09030 .0751]^{\mathrm{T}} ; \lambda \max =10.5354 ; C R=0.0399$

The relative importance weights $W$ of judgment matrix C2-D and $\lambda$ max, CR: $W=[0.77590 .21470 .4459$ $0.12320 .22190 .18370 .13500 .12590 .11100 .0920]^{\mathrm{T}} ; \lambda \max =10.7535 ; C R=0.0562$

The relative importance weights $W$ of judgment matrix C3-D and $\lambda \max , \mathrm{CR}: W=[0.79950 .13740 .4221$ $0.19950 .18930 .20250 .12340 .12340 .10170 .0797]^{\mathrm{T}} ; \lambda \max =10.3838 ; C R=0.0286$

The relative importance weights $W$ of judgment matrix C4-D and $\lambda$ max, CR: $W=[0.81060 .15150 .4064$ $0.20080 .19440 .19440 .11850 .11590 .08390 .0664]^{\mathrm{T}} ; \lambda \max =10.4248 ; C R=0.0317$

The relative importance weights $W$ of judgment matrix C5-D and $\lambda \max , \mathrm{CR}: W=[0.78860 .11500 .4491$ $0.19240 .20060 .20060 .12220 .12220 .09310 .0844]^{\mathrm{T}} ; \lambda \max =10.2648 ; C R=0.0197$

The relative importance weights $W$ of judgment matrix C6-D and $\lambda$ max, CR: $W=[0.76150 .12890 .4114$ $0.24200 .24250 .24250 .14590 .14590 .10040 .0739]^{\mathrm{T}} ; \lambda \max =10.2560 ; C R=0.0191$

The relative importance weights $W$ of judgment matrix C7-D and $\lambda$ max, CR: $W=[0.74190 .12720 .4742$ $0.21520 .27370 .20210 .15000 .11800 .07690 .0640]^{\mathrm{T}} ; \lambda \max =10.4464 ; C R=0.0333$

4). Consistency check

In order to analyze the rationality and reliability of the weight distribution, we seek general index $C_{R a}$ of judgment coefficient matrix $A_{a}$ by the method of using literature [13] to determine consistency check of the coefficient matrix $A_{a}$. When $C_{R a}$ is less than 0.1 , it is considered that the judgment matrix $A_{i}$ with optimum consistency, indicating that empower is reasonable; Otherwise, we need to adjust the coefficient matrix Ai, until a satisfactory consistency so far.

\section{The total level sorting}

Table 1 total sequencing calculation table

\begin{tabular}{|c|c|c|c|c|c|c|c|c|c|}
\hline \multirow{3}{*}{$\begin{array}{l}\text { rank } \\
\text { layer B to } \\
\text { layer A }\end{array}$} & \multicolumn{3}{|c|}{ B1 } & \multicolumn{2}{|c|}{ B2 } & \multicolumn{2}{|c|}{ B3 } & \multirow{6}{*}{$\begin{array}{l}\text { the } \\
\text { total } \\
\text { sorting } \\
\text { weight } \\
\text { of D } \\
\text { layer }\end{array}$} & \multirow{6}{*}{ number } \\
\hline & \multirow{2}{*}{\multicolumn{3}{|c|}{$\frac{1}{0.9161}$}} & \multicolumn{2}{|c|}{2} & \multicolumn{2}{|c|}{3} & & \\
\hline & & & & \multicolumn{2}{|c|}{0.3715} & \multicolumn{2}{|c|}{0.1506} & & \\
\hline \multirow{3}{*}{\begin{tabular}{|l}
\multicolumn{1}{c}{ rank } \\
layer C \\
to \\
layer \\
rank \\
layer \\
D to \\
layer C
\end{tabular}} & $\mathrm{C} 1$ & $\mathrm{C} 2$ & C3 & $\mathrm{C} 4$ & C5 & C6 & C7 & & \\
\hline & 1 & 2 & 3 & 1 & 2 & 1 & 2 & & \\
\hline & 0.8021 & 0.5053 & 0.3183 & 0.8944 & 0.4472 & 0.8944 & 0.4472 & & \\
\hline D1 & 0.7657 & 0.7759 & 0.7995 & 0.8106 & 0.7886 & 0.7615 & 0.7419 & 3.3513 & 1 \\
\hline D2 & 0.2348 & 0.2147 & 0.1374 & 0.1515 & 0.1150 & 0.1289 & 0.1272 & 0.6997 & 6 \\
\hline D3 & 0.4815 & 0.4459 & 0.4221 & 0.4064 & 0.4491 & 0.4114 & 0.4742 & 1.8902 & 2 \\
\hline D4 & 0.1477 & 0.1232 & 0.1995 & 0.2008 & 0.1924 & 0.2420 & 0.2152 & 0.8226 & 5 \\
\hline D5 & 0.1905 & 0.2219 & 0.1893 & 0.1944 & 0.2006 & 0.2425 & 0.2737 & 0.9280 & 3 \\
\hline D6 & 0.1658 & 0.1837 & 0.2025 & 0.1944 & 0.2006 & 0.2425 & 0.2021 & 0.8610 & 4 \\
\hline D7 & 0.1171 & 0.1350 & 0.1234 & 0.1185 & 0.1222 & 0.1459 & 0.1500 & 0.5596 & 7 \\
\hline D8 & 0.1171 & 0.1259 & 0.1234 & 0.1159 & 0.1222 & 0.1459 & 0.1180 & 0.5384 & 8 \\
\hline D9 & 0.0903 & 0.1110 & 0.1017 & 0.0839 & 0.0931 & 0.1004 & 0.0769 & 0.4017 & 9 \\
\hline D10 & 0.0751 & 0.0920 & 0.0797 & 0.0664 & 0.0844 & 0.0739 & 0.0640 & 0.3239 & 10 \\
\hline
\end{tabular}


Calculate sort weights of all the factors of the relative importance for the uppermost on the same level. All factors layer by layer from the top to the lowest sort, programs relative to the total level of the overall goal sorting calculated is shown in Table 1.

\section{Risk assessment of AHP}

1) Establishment of weight set:

The weight of corresponding factors of elements by calculating AHP:

$W=\left[\begin{array}{lllllllllllll}3.3513 & 0.6997 & 1.8902 & 0.8226 & 0.9280 & 0.8610 & 0.5596 & 0.5384 & 0.4017 & 0.3239\end{array}\right]^{\mathrm{T}}$

2) Establishment of reviews set:

$V=$ [great risk, larger risk, risk in general, smaller risk, minimal risk]

3) Fuzzy evaluation of univariate factor:

After a summary of the questionnaire feedback, we can get

$$
I=\left[\begin{array}{lllll}
0.60 & 0.82 & 0.43 & 0.12 & 0.08 \\
0.42 & 0.54 & 0.75 & 0.10 & 0.12 \\
0.56 & 0.68 & 0.38 & 0.13 & 0.19 \\
0.38 & 0.42 & 0.65 & 0.25 & 0.11 \\
0.35 & 0.56 & 0.73 & 0.32 & 0.13 \\
0.30 & 0.52 & 0.52 & 0.30 & 0.16 \\
0.26 & 0.48 & 0.47 & 0.41 & 0.14 \\
0.22 & 0.36 & 0.40 & 0.40 & 0.15 \\
0.20 & 0.23 & 0.37 & 0.25 & 0.18 \\
0.18 & 0.16 & 0.28 & 0.10 & 0.26
\end{array}\right]
$$

4) Fuzzy Comprehensive Evaluation: $M=W^{T} \cdot I=[4.6615$ 6.3309 5.1306 2.0187 1.1867]

According to the evaluation results, we can know that enterprise finance have the greater risk.

\section{Conclusion}

This paper evaluates enterprise financing risk based on analytic hierarchy process, which takes advantage of the expertise to achieve a qualitative analysis and quantitative assessment of risk. The findings of the study are shown as follows: firstly, legal restrictions and national macro-control policies have the maximum impact on corporate finance. Chinese enterprise financing mainly through bank loans, public financing and debt financing are subject to national and local policies and laws largely. Secondly, monopoly and competition between the industry and industry threshold limit have large impact on enterprise financing. Finally, corporate profit ability, operation ability, debt paying ability and enterprise scale has little effect on the financing risks.

\section{Acknowledgements}

This paper is sponsored by Hubei province department of education Humanities and Social Science Research Project: Financial Crisis Shock, Financing Constraints and Corporate Value: Theoretical Exploration and Empirical Research (14D004); Social science fund of Hubei province: Financial Development and Current Account Sustainability: Theoretical Exploration and Empirical Research (2013129).

\section{References}

[1] Jianpu Wang, Financing risks for Chinese enterprise in the international operation [J]. Reformation\&Strategy, 2011, 27(6):142-144. 
[2] Xiangjuan Tang, Forms and Prevention Strategy Analysis of Financing Risk of Small and Medium-size Enterprises [C]. 2012 International Conference on Education Technology and Management Engineering (ETME 2012) 98-102.

[3] Yede Huang, Lixia Jia. A Research of Financing Risk Management in Small and Medium-sized Enterprises [C]. Proceedings of the Conference on Web Based Business Management,2010:500-504.

[4] Myers S C, Majluf N S. Corporate Financing and Investment Decisions When Firms Have Information that Investors Do Not Have [J]. Journal of financial economics, 1984, 13(2): 187-221.

[5] Anping Zhang, Information Asymmetry: Theoretical analysis and policy path of finance risk on small and medium foreign enterprises[J].Journal of Finance and Economics ,2010(10):22-25.

[6] Xiumin Du, The analysis of financing method and financing risk on small and medium enterprises [J]. China Business\&Trade,2010(16):86-87.

[7] Yanlin Wang, Empirical analysis of financing risks on small and medium enterprises - test data from small board listed companies [J].Communication of Finance and Acounting,2012(10):117-119.

[8] Suhong Li,Liwen Chen,Shuqiang Wang, Financing risk evaluation based on fuzzy analytic hierarchy process - for example real estate development enterprises in Hebei [J].Enterprise Economy,2013(2):137-140.

[9] Li X, Zhang R. The Risk Assessment of Logistic Finance Based on the Fuzzy Analytic Hierarchy Process [M]//LISS 2012. Springer Berlin Heidelberg, 2013: 421-425.

[10] Xiaojun Fan,Fanghua Wang,Yinyuan Zhong, Dynamic Fuzzy Evaluation of financing risk on large-scale infrastructure project [J]. Journal of Shanghai Jiaotong University,2004, 38(3):450-454.

[11] JieGuo, Yajie Dong, Case studies of corporate finance risk based on efficacy coefficient method [J]. Friends of Accounting,2009(28):27-29.

[12] Fabin Zhao,Application of Analytic Hierarchy Process in Risk Management - A Case Study of training equipment financing risks [J]. Journal of Kunming Metallurgy College,2013(1):67-72.

[13] Shubo Xu, Analytic Hierarchy Process Principle [M]. Tianjin:Tianjin University Press,1998. 